OPEN ACCESS

Edited by:

Hui Wu,

East China University of Science and

Technology, China

Reviewed by:

Guang Zhao,

Shandong University, China

Brian Pfleger,

University of Wisconsin-Madison,

United States

*Correspondence:

Xiaochao Xiong

xcxiong@wsu.edu

Specialty section:

This article was submitted to

Microbial Physiology and Metabolism, a section of the journal

Frontiers in Microbiology

Received: 21 March 2020

Accepted: 30 June 2020

Published: 24 July 2020

Citation:

Ghogare $R$, Chen $S$ and Xiong $X$

(2020) Metabolic Engineering of

Oleaginous Yeast Yarrowia lipolytica for Overproduction of Fatty Acids.

Front. Microbiol. 11:1717.

doi: 10.3389/fmicb.2020.01717

\section{Metabolic Engineering of Oleaginous Yeast Yarrowia lipolytica for Overproduction of Fatty Acids}

\author{
Rishikesh Ghogare, Shulin Chen and Xiaochao Xiong* \\ Department of Biological Systems Engineering, Washington State University, Pullman, WA, United States
}

The oleaginous yeast Yarrowia lipolytica has attracted much attention due to its ability to utilize a wide range of substrates to accumulate high lipid content and its flexibility for genetic manipulation. In this study, intracellular lipid metabolism in Y. lipolytica was tailored to produce fatty acid, a renewable oleochemical and precursor for production of advanced biofuels. Two main strategies, including blocking activation and peroxisomal uptake of fatty acids and elimination of biosynthesis of lipids, were employed to reduce fatty acid consumption by the native pathways in Y. lipolytica. Both genetic modifications improved fatty acid production. However, disruption of the genes responsible for assembly of nonpolar lipid molecules including triacylglycerols (TAGs) and steryl esters resulted in the deleterious effects on the cell growth. The gene tes $A$ encoding thioesterase from Escherichia coli was expressed in the strain with disrupted faa genes encoding fatty acyl-CoA synthetases and pxa1 encoding peroxisomal acyl-CoA transporter, and the titer of fatty acids resulted in $2.3 \mathrm{~g} / \mathrm{L}$ in shake flask culture, representing 11-fold improvement compared with the parent strain. Expressing the native genes encoding acetyl-CoA carboxylase (ACC) and hexokinase also increased fatty acid production, although the improvement was not as significant as that with tes $A$ expression. Saturated fatty acids including palmitic acid (C16:0) and stearic acid (C18:0) increased remarkably in the fatty acid composition of the recombinant bearing tesA compared with the parent strain. The recombinant expressing tes $A$ gene resulted in high lipid content, indicating the great fatty acid producing potential of Y. lipolytica. The results highlight the achievement of fatty acid overproduction without adverse effect on growth of the strain. Results of this study provided insight into the relationship between fatty acid and lipid metabolism in Y. lipolytica, confirming the avenue to reprogram lipid metabolism of this host for overproduction of renewable fatty acids.

Keywords: Yarrowia lipolytica, fatty acid, oleaginous yeast, fatty acyl-CoA, thioesterase, lipid

\section{INTRODUCTION}

Increase in greenhouse gas emissions and volatility in fossil fuel prices have prompted development of alternative chemicals and fuels produced by microorganisms from renewable biomass. Microbial lipids produced from renewable feedstock such as cellulosic sugars can be used as the precursors for sustainable production of fungible fuels including green diesel and biodiesel with high-energy density (Xiong et al., 2012). The model organisms such as Escherichia coli and Saccharomyces cerevisiae have been intensively engineered for production of lipids and lipid-derived chemicals (Steen et al., 2010). 
Compared with E. coli and S. cerevisiae (Ferreira et al., 2018), oleaginous yeasts have superior natural ability to accumulate large amount of lipids, mainly consisting of triacylglycerols (TAGs; Adrio, 2017). Among these oleaginous strains, Yarrowia lipolytica is one of the most intensively studied non-conventional yeasts and serves as a model for investigating the mechanism of lipid accumulation (Madzak, 2018). Because of its generally recognized as safe (GRAS) status, Y. lipolytica has been metabolically engineered for production of an array of renewable chemicals such as succinic acid (Cui et al., 2017) and triacetic acid lactone (TAL; Markham et al., 2018) and other high-value bioproducts such as $\beta$-carotene and limonene (Cao et al., 2016).

In Y. lipolytica, TAGs are the main storage forms of fatty acids, and metabolism of fatty acid plays an important role in production and accumulation of lipids (Beopoulos et al., 2012). Fatty acids themselves are valuable oleochemical, and they can be readily converted into fuels such as biodiesel and alkane and other chemicals such as fatty alcohols and long-chain dicarboxylic acids by either chemical or biological means (Steen et al., 2010). In oleaginous strains, TAGs are often stored in the cells as lipid droplets (Gajdoš et al., 2016). However, fatty acids can be secreted out by some strains due to the straightchain structures of fatty acid molecules (Leber et al., 2015). It is naturally to believe that $Y$. lipolytica can be designed and engineered as a cell factory for efficient production of fatty acids by tailoring intracellular lipid metabolism.

Metabolic engineering strategies have been used to improve production of lipids in Y. lipolytica, including overexpressing gene(s) for improvement of precursors supply for fatty acid biosynthesis ("push"), overexpressing genes for fatty acid formation and TAG assembly ("pull"), and deleting genes for $\beta$-oxidation ("block"; Liu et al., 2017). Overexpression of two genes, YlAcc1 and YlDga1, encoding the enzymes, acetyl-CoA carboxylase (ACC) and diacylglycerol acyltransferase (DGAT) increased lipid content to $61.7 \%$ of dry cell weight (DCW), with lipid yield of $0.195 \mathrm{~g} / \mathrm{g}$ glucose and productivity of $0.143 \mathrm{~g} / \mathrm{L} / \mathrm{h}$ achieved by Y. lipolytica (Tai and Stephanopoulos, 2013). Lipid titers of 39.1 and $55 \mathrm{~g} / \mathrm{L}$ with maximal lipid productivities of 0.89 and $1 \mathrm{~g} / \mathrm{L} / \mathrm{h}$ have been achieved with evolutionary and rational engineering, respectively (Liu et al., 2015). In Y. lipolytica, overexpression of the native gene YlHxkl encoding hexokinase enhanced cell growth, reduced filament formation, and improved lipid content when grown on hexose sugars such as glucose and fructose. By engineering NADPH regeneration pathway in Y. lipolytica, the strain achieved a productivity of $1.2 \mathrm{~g} / \mathrm{L} / \mathrm{h}$ for lipid accumulation (Qiao et al., 2017). To engineer the strains for production of fatty acids, some of these strategies including improvement of fatty acid biosynthesis and deleting genes for $\beta$-oxidation can be adopted because TAG formation is dependent on incorporation of fatty acids as major backbone. However, to develop strains for more efficient production of fatty acids, it is critical to specifically engineer the metabolism of fatty acid based on the understanding of the mechanism underlying formation, activation, utilization, and degradation of fatty acids in the cells.

Considerable efforts have already been made to metabolically engineer the model organism S. cerevisiae for overproduction of fatty acids. In the yeast cells such as $S$. cerevisiae, there are cellular compartments to isolate metabolites. Long-chain fatty acids $(\mathrm{C} 16-\mathrm{C} 18)$ are mainly degraded through $\beta$-oxidation in the organelle, peroxisome. Prior to $\beta$-oxidation, fatty acids are activated to fatty acyl-CoA and then transported by $\mathrm{ABC}$ transporters into peroxisome (Leber et al., 2015). In S. cerevisiae, peroxisomal transporter complex PXA1-2 plays an important role in transporting acyl-CoA into peroxisome (Leber et al., 2015). There are four fatty acyl-CoA synthetases encoded by faa genes, namely, ScFaa1,ScFaa2, ScFaa3, and ScFaa4 in S. cerevisiae (Faergeman et al., 2001). In addition, there are two fatty acid transporters ScFatl, which is responsible for uptake and transport of long-chain fatty acids, and ScFat2 with unknown function (Coe et al., 1999). Studies suggest that ScFaal and ScFaa4 play a major role in activation of free fatty acids in S. cerevisiae. The deletion of ScFaa1 and ScFaa4 led to production of $310 \mathrm{mg} / \mathrm{L}$ of intracellular and $320 \mathrm{mg} / \mathrm{L}$ extracellular fatty acids, and the yield of fatty acids was further improved by blocking $\beta$-oxidation (Leber et al., 2015). Moreover, heterologous expression of thioesterase from Mus musculus improved fatty acid production and secretion by S. cerevisiae (Chen et al., 2014). The elimination of biosynthesis of TAGs and steryl esters in S. cerevisiae also increased fatty acid production (Sandager et al., 2002; Valle-Rodríguez et al., 2014). In addition to deletion of Faa1, Faa4, and Pox1 gene encoding fatty acyl-CoA oxidase and expression of tes $A$ encoding a truncated $E$. coli thioesterase, a hybrid pathway consisting of ATP citrate lyase (ACL) from M. musculus, malic enzyme (ME) from Rhodosporidium toruloides, and endogenous malate dehydrogenase (Mdh3) with eliminated peroxisomal signal peptide was constructed to enhance generation of acetyl-CoA, and the resultant $S$. cerevisiae strain could produce $10.4 \mathrm{~g} / \mathrm{L}$ of $\mathrm{C} 16-\mathrm{C} 18$ fatty acids in fed-batch fermentation (Zhou et al., 2016). By combination of metabolic engineering and adaptive laboratory evolution, a Crabtree negative S. cerevisiae without ethanol production was developed and further improvement led to production of $33.4 \mathrm{~g} / \mathrm{L}$ free fatty acids (Dai et al., 2018).

In addition to the model organism $S$. cerevisiae, fatty acid metabolism was investigated in Y. lipolytica, and the finding of these studies could provide insights to design and engineer efficient cell factories (Figure 1). In Y. lipolytica, there were two genes, YlPxa1 (YALI0A06655g) and YlPxa2 (YALI0D04246g), with identities to their corresponding genes in S. cerevisiae, which were responsible for the transport of fatty acyl-CoA into peroxisome (Dulermo et al., 2015; Leber et al., 2015). Knockout of YlFaal (YALIOD17864g) increased the contents of both total lipids and saturated fatty acids (Wang et al., 2011). In Y. lipolytica, YlFat 1 had a different function than ScFat1, and it was involved in mobilization of fatty acids from lipid droplets after the hydrolysis of TAGs catalyzed by lipase (Dulermo et al., 2014). However, the other possible faa genes encoding fatty acyl-CoA synthetase in Y. lipolytica have been neither reported nor targeted for metabolic engineering. As an oleaginous organism, Y. lipolytica accumulates high TAG levels. In the final step of assembly, TAGs are either formed by combination of diacylglycerol (DAG) with acyl-CoA, which is catalyzed by DGAT encoded with genes YlDgal (YALI0E32769g) and YlDga2 (YALI0D07986g), or acyl-CoA independent pathway mediated with phospholipid:diacylglycerol acyltransferase (PDAT) encoded by YlLro1 (YALI0E16797g; 


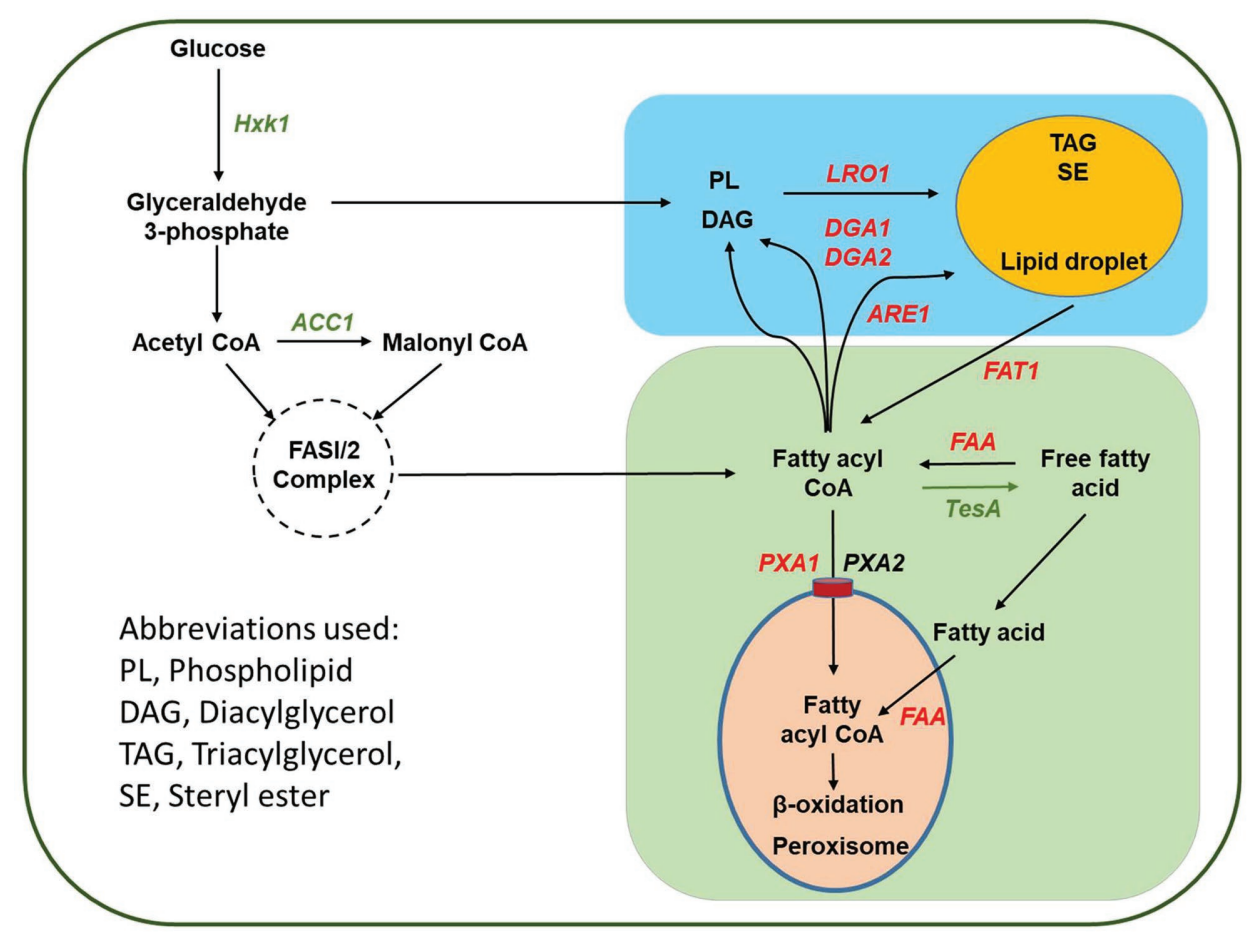

FIGURE 1 | Schematic representation of the pathway design for metabolic engineering of Yarrowia lipolytica to produce free fatty acid. The genes to be deleted are shown with red font, whereas the gene targets for overexpression are shown with green font.

Tai and Stephanopoulos, 2013; Figure 1). In lipid droplets, there are also steryl esters. In Y. lipolytica, biosynthesis of steryl esters is catalyzed by steryl ester synthase, which is encoded by gene of YlAre1 (YALI0F06578g), belonging to the acyl-CoA cholesterol acyltransferase family (Koch et al., 2014). Overproduction of fatty acids was achieved by metabolic engineering of Y. lipolytica through the combinatorial deletion of one fatty acyl-CoA synthetase gene and elimination of $\beta$-oxidation and additional overexpression of intracellular lipases for hydrolysis of TAGs into fatty acids (Ledesma-Amaro et al., 2016).

To develop a highly productive strain for production of fatty acids, it requires optimization of multiple metabolic modules, including increasing acetyl-CoA supply and malonyl-CoA generation, balancing cofactor NADPH, blocking fatty acid degradation, improving fatty acid production, and controlling conversion of fatty acids into lipids in a systematic way (Yan and Pfleger, 2020). This study employed two different strategies, which included blocking activation and peroxisomal uptake of fatty acids and elimination of biosynthesis of TAGs and steryl esters, to limit fatty acid consumption in Y. lipolytica. Multiple candidate faa genes were identified and disrupted in the host. Due to better performance of cell growth and fatty acid production, the strain with disrupted genes of fatty acyl-CoA synthetases and peroxisomal acyl-CoA transporter was selected for next-step engineering. Fatty acid production was further improved by expressing native genes, YlAcc1, YlHxk1, and TesA gene from E. coli in the mutant strain. Expression of tes $A$ gene led to great increase in fatty acid production. The results highlight the achievement of fatty acid overproduction without detrimental effects on cell growth, existence of multiple faa genes in Y. lipolytica, and the importance of biosynthesis of non-polar lipid molecules, although they were previously believed as non-essential pathways in S. cerevisiae.

\section{MATERIALS AND METHODS}

\section{Strains and Culture Conditions}

E. coli TOP10 was used as the host strain for cloning of genes and propagation of plasmids (Table 1). E. coli carrying plasmids was grown at $37^{\circ} \mathrm{C}$ on lysogeny broth (LB) medium supplemented with $100 \mu \mathrm{g} / \mathrm{ml}$ ampicillin (Xiong et al., 2016). The yeast strains were derived from Y. lipolytica Po4f, a Ku70-deleted strain of PO1f (ATCC MYA-2613) that was auxotrophic for both uracil and leucine (Wang et al., 2016). Y. lipolytica strains were cultivated in flasks with yeast extract peptone dextrose (YPD) media consisting of $10 \mathrm{~g} / \mathrm{L}$ of yeast extract (Difco), $20 \mathrm{~g} / \mathrm{L}$ of peptone (Difco), and $20 \mathrm{~g} / \mathrm{L}$ of glucose at $28^{\circ} \mathrm{C}$ on a shaker with a shaking speed of $180 \mathrm{rpm}$. YPD agar plates were made by adding $15 \mathrm{~g} / \mathrm{L}$ agar (Difco). Yeast transformants were grown in selective media depending on genotype. The yeast transformants were selected and isolated on agar plates of synthetic media lacking either uracil or leucine. Selective media was composed of $20 \mathrm{~g} / \mathrm{L}$ glucose and $6.7 \mathrm{~g} / \mathrm{L}$ yeast nitrogen base YNB without amino acid and with ammonium sulfate (US Biologicals), supplemented with $2.0 \mathrm{~g} / \mathrm{L}$ of complete supplement of amino acids lacking uracil or leucine (US Biologicals). Agar plates of selective media were prepared by adding $15 \mathrm{~g} / \mathrm{L}$ agar to broth. 
The media for growing and testing the cells for production of fatty acids contained $80 \mathrm{~g} / \mathrm{L}$ glucose, $20 \mathrm{~g} / \mathrm{L}$ yeast extract, $1.36 \mathrm{~g} / \mathrm{L}$ ammonium sulfate, and $1.7 \mathrm{~g} / \mathrm{L}$ yeast nitrogen base without amino acid and ammonium sulfate. The cultivation was carried out by inoculating single colonies into culture tubes containing $3 \mathrm{ml}$ media and then incubating at $28^{\circ} \mathrm{C}$ and $180 \mathrm{rpm}$ for $48 \mathrm{~h}$. The seed cultures were then used to inoculate $50 \mathrm{ml}$ of media in a $250 \mathrm{ml}$ baffled flask, which was cultivated at $28^{\circ} \mathrm{C}$ and $180 \mathrm{rpm}$. The initial absorbance at $600 \mathrm{~nm}\left(\mathrm{OD}_{600}\right)$ of the culture was adjusted to 0.05 .

\section{DNA Techniques}

Plasmid DNA was extracted from E. coli by using a GeneJET Plasmid Miniprep Kit from Thermo Fisher Scientific. FastDigest restriction endonucleases were purchased from Thermo Fisher Scientific. To get DNA fragments for cloning, PCR was performed with Q5 high-fidelity DNA polymerase from NEB. Other PCR was routinely carried out by using DreamTaq Green PCR Master Mix from Thermo Scientific. Oligonucleotide primers were synthesized in Invitrogen (Grand Island, NY). The sequence and generated restriction sites of the primers are listed in Supplementary Table S1. The PCR products and plasmids digested by restriction endonucleases were recovered from agarose gel after electrophoresis with GeneJET Gel Extraction kit (Thermo Scientific). The competent cells of $E$. coli were made by $\mathrm{CaCl}_{2}$ solution and transformed with DNA by fowling the heat-shock procedure. Prior to transformation of Y. lipolytica, the digested DNA fragments were recovered by using DNA Clean \& Concentrator kit (Zymo Research, Irvine, CA). Y. lipolytica cell was transformed with linearized plasmid or DNA fragment by using Frozen-EZ Yeast Transformation II Kit (Zymo Research, Irvine, CA).

\section{Construction of Recombinant Strains for Overproducing Fatty Acids}

The gene encoding Ku70 in Y. lipolytica Polf was disrupted to enhance the homologous recombinant frequency, and new strain was designated Po4f. Around 1.0-kb DNA fragments of $5^{\prime}$ and $3^{\prime}$ region of targeted genes to be deleted were amplified with primers (Supplementary Table S1) by PCR and then cloned into the plasmid containing selection marker gene ura3. To knockout a set of selected genes in Y. lipolytica Po4f, the procedure of the homologous recombination was followed, including Cre/loxP-mediated excision of the selection marker ura3 and curing of the plasmid pJN44-cre carrying the Cre gene encoding recombinase.

The 7.27-kb YlAcc1 gene containing intron was amplified by PCR with primers YlAcc1-F and YlAcc1-R using Y. lipolytica genome DNA as template. After digestion of PCR product with SmaI, YlAccl gene was inserted into expression vector pGR12 developed previously (Wang et al., 2016). In the resultant plasmid pGR12 Acc1, expression of the native gene Acc1 from $Y$. lipolytica was under control of FBA promoter. Similarly, the plasmid pGR52 Hxk was constructed to express native gene YlHxk1 driven by GPM promoter (Wang et al., 2016). A new vector pJN44 TesA containing TEF promoter with first intron (TEFIN) was developed to express $0.55-\mathrm{kb}$ tes $A$ gene encoding leaderless thioesterase I from E. coli K12. The vector pGR12 with leu 2 gene as a selection marker was treated by restriction enzyme SpeI. The linearized pGR12 was integrated into Y. lipolytica Po4f and YlRX. The restriction enzyme SpeI was used to linearize plasmids pGR12 Acc1 and pGR52 Hxk, whereas plasmid pJN44 TesA was digested with SphI. The DNA fragments containing both leu2 marker and expression cassettes of cloned genes were integrated into the genome of knockout strain YIRX by transformation with linearized plasmids. Transformants were cultured and isolated on the synthetic agar plates without leucine. The strains used in this study are listed in Table $\mathbf{1 .}$

\section{Quantification of Lipids and Free Fatty Acids by Gas Chromatography Analysis}

Extraction and transesterification of lipids were performed using the method described previously (Xiong et al., 2012). Extracted fatty acid methyl esters (FAMEs) in hexane were used for gas chromatography (GC) analysis with tridecanoic acid (C13:0) as an internal standard. The protocol for extraction of free fatty acids was developed by modifying the lipid extraction method developed by Folch et al. (1957). Cell culture with volume of $0.5 \mathrm{ml}$ was mixed with $0.75 \mathrm{ml}$ chloroform: methanol (2:1) solution. Cells were lysed by using a Mini-BeadBeater with $0.5 \mathrm{~mm}$ glass beads (BioSpec Products, Inc., Bartlesville, OK). The mixture was centrifuged at $13,000 \mathrm{rpm}$ for $10 \mathrm{~min}$ to

TABLE 1 | Strains used in this study.

\begin{tabular}{|c|c|c|}
\hline Strain & Characteristic(s) & $\begin{array}{l}\text { Reference or } \\
\text { source }\end{array}$ \\
\hline E. coli Top10 & $\begin{array}{l}\mathrm{F}^{-} \text {mcrA } \Delta(\text { mrr-hsdRMS-mcrBC) } \\
\varphi 80 l a c Z \Delta \mathrm{M} 15 \Delta \text { lacX74 recA1 } \\
\text { araD139 } \Delta \text { (ara leu) } 7697 \text { ga/U ga/K } \\
\text { rpsL (StrR) endA1 nupG }\end{array}$ & Invitrogen \\
\hline E. coli $\mathrm{K} 12$ & Wild type, source of tesA & $\begin{array}{l}\text { E. coli Genetic Stock } \\
\text { Center (CGSC) }\end{array}$ \\
\hline Y. lipolytica Po1f & $\begin{array}{l}\text { MatA, leu2-270, ura3-302, } \\
\text { xpr2-322, axp1-2 }\end{array}$ & ATCC MYA-2613 \\
\hline Y. lipolytica Po4f & Ku70 knockout, leu2- , ura3- & (Wang et al., 2016) \\
\hline Y. lipolytica YIR1 & $\begin{array}{l}\text { Y. lipolytica Po4f penta knockout } \\
\triangle \text { Faa1, } \triangle \text { Faa2, } \triangle \text { Faa3, } \triangle \text { Fat1, } \\
\triangle \text { Pxa1, leu2 }{ }^{-}, \text {ura3 }^{+}\end{array}$ & This work \\
\hline Y. lipolytica YIR2 & $\begin{array}{l}\text { Y. lipolytica Po4f quadra knockout } \\
\Delta \text { Dga1, } \Delta \text { Dga2, } \Delta \text { Lro1, } \triangle \text { Are1, } \\
\text { leu2 } 2^{-}, \text {ura3 }^{+}\end{array}$ & This work \\
\hline Y. lipolytica Po4f Ura & Y. lipolytica Po4f, leu2-, ura3+ & This work \\
\hline Y. lipolytica YIRX & $\begin{array}{l}\text { Y. lipolytica Po4f penta knockout } \\
\triangle \text { Faa1, } \triangle \text { Faa2, } \triangle \text { Faa3, } \triangle \text { Fat1, } \\
\triangle \text { Pxa1, leu2 }{ }^{-}, \text {ura3 }^{-}\end{array}$ & This work \\
\hline $\begin{array}{l}\text { Y. lipolytica Po4f } \\
\text { Control }\end{array}$ & $\begin{array}{l}\text { Y. lipolytica Po4f integrated with } \\
\text { linearized plasmid pGR12, leu2+, } \\
\text { ura3- }^{+}\end{array}$ & This work \\
\hline $\begin{array}{l}\text { Y. lipolytica YIRX } \\
\text { Control }\end{array}$ & $\begin{array}{l}\text { Y. lipolytica YIRX integrated with } \\
\text { linearized plasmid pGR12, leu2+, } \\
\text { ura3- }^{+}\end{array}$ & This work \\
\hline $\begin{array}{l}\text { Y. lipolytica YIRX } \\
\text { Acc1 }\end{array}$ & $\begin{array}{l}\text { Y. lipolytica YIRX overexpressing } \\
\text { Ylacc1, leu2 }^{+} \text {, ura3- }\end{array}$ & This work \\
\hline $\begin{array}{l}\text { Y. lipolytica YIRX } \\
\text { Hexo }\end{array}$ & $\begin{array}{l}\text { Y. lipolytica YIRX overexpressing } \\
\text { YIHXk1, leu2 }{ }^{+}, \text {ura3 }^{-}\end{array}$ & This work \\
\hline $\begin{array}{l}\text { Y. lipolytica YIRX } \\
\text { TesA }\end{array}$ & $\begin{array}{l}\text { Y. lipolytica YIRX overexpressing } \\
\text { tesA, leu2 } 2^{+}, \text {ura3 }^{-}\end{array}$ & This work \\
\hline
\end{tabular}


separate the organic phase, and then the organic phase was washed with $0.9 \% \mathrm{NaCl}$ solution. The organic phase containing fatty acids was directly used for analysis with GC. GC analysis was performed using Agilent 7890A GC (Agilent Technologies, Inc., Santa Clara, CA) equipped with a flame ionization detector (FID) and FAMEWAX column $(30 \mathrm{~m} \times 320 \mu \mathrm{m} \times 0.25 \mu \mathrm{m}$; Restek Corporation). One microliter of the sample was injected with a split ratio of $20: 1$. Injector temperature was set at $250^{\circ} \mathrm{C}$, and the initial oven temperature was set at $120^{\circ} \mathrm{C}$, ramped up at the rate of $5^{\circ} \mathrm{C} / \mathrm{min}$ to $240^{\circ} \mathrm{C}$, and then held for $25 \mathrm{~min}$. The detector temperature was set at $250^{\circ} \mathrm{C}$. Fatty acids produced by $Y$. lipolytica were quantified by using the standard curves of individual fatty acids with different contents (Sigma).

\section{Measurement of Glucose Content, Cell Dry Weight, and Cell Growth}

Samples were collected every $24 \mathrm{~h}$ for measurement of residual glucose. One milliliter culture was centrifuged at 13,000 rpm. Supernatant was used for determination of residual glucose in the medium. Glucose was analyzed using Dionex ICS-3000 Ion Chromatography equipped with CarboPac TM PA20 analytical column and CarboPac TM PA20 guard column (Xiong et al., 2016). Glucose concentration was quantified by using the external standard method.

The cell growth was determined by measuring the absorbance at $600 \mathrm{~nm}$ of the culture using Shimadzu UV-Vis spectrophotometer, UV-2550. For cell dry weight (CDW) measurement, $5 \mathrm{ml}$ of cell culture was collected and centrifuged at $13,000 \mathrm{rpm}$ for $10 \mathrm{~min}$. The pellets were washed twice with $5 \mathrm{ml}$ of distilled water and dried at $104^{\circ} \mathrm{C}$, until a consistent weight was obtained (approximately $24 \mathrm{~h}$ ). The data of weight of dried cell biomass were recorded.

\section{RESULTS}

\section{Development of Knockout Strains for Producing Fatty Acids}

By using the sequences of faa genes from S. cerevisiae as the reference, four candidate genes, including YlFaal (YALI0D17864g), YlFaa2 (YALIOE12859g), YlFaa3 (YALIOF06556g), and YlFat1 (YALI0E16016g), have been identified for activation of fatty acids into acyl-CoA in Y. lipolytica (Leber et al., 2015). All the enzymes encoded by these four genes from $Y$. lipolytica have potential adenosine monophosphate (AMP) binding domains, one of the important characteristics of fatty acyl-CoA synthetases (Shockey et al., 2000), and YlFaal and Fat1 genes have been identified and studied previously (Wang et al., 2011; Dulermo et al., 2014). The identities of faa genes from Y. lipolytica with the corresponding genes from $S$. cerevisiae were summarized in Supplementary Table S2. The protein sequence of peroxisomal ABC transporter YlPxal (YALI0A06655g) showed around 40\% identity with ScPxal (Dulermo et al., 2015). These genes were targeted for deletion to impair fatty acid activation and transport of fatty acyl-CoA to peroxisome for $\beta$-oxidation in Y. lipolytica (Figure 1). Mutant Y. lipolytica YlR1 ( $\triangle F a a 1, \Delta F a a 2, \Delta F a a 3, \Delta F a t 1$, and $\Delta$ Pxa1) was constructed by combinatorial deletion of the genes encoding fatty
acyl-CoA synthetases and a peroxisomal transporter (Table 1). To investigate the relationship between biosynthesis of lipid molecules including TAGs and steryl esters and fatty acid production, a mutant strain Y. lipolytica YlR2 ( $\triangle$ YlDga1, $\Delta$ YlDga2, $\triangle Y l L r o 1$, and $\triangle Y l$ Are 1) bearing multiple disrupted genes involved in lipid assembly was constructed (Table 1).

\section{Production of Fatty Acids by Y. lipolytica Knockouts}

The results of fatty acid content measurement indicated that the mutant $Y$. lipolytica YlR1 could accumulate more fatty acids than both strain YlR2 and the control, Po4f Ura (Figure 2A). The titer of fatty acids produced by Y. lipolytica YlR1 reached the highest value $(0.755 \mathrm{~g} / \mathrm{L})$ on day 5 and kept this similar level for the last 3 days. The fatty acid concentration in Y. lipolytica YlR1 was three times over that of YlR2 (Figure 2A). The results show that deletion of genes responsible for fatty acid activation and peroxisomal transport successfully improved fatty acid production by Y. lipolytica, but the mutant YlR2 showed no remarkable increase in fatty acid titer. The highest concentration of fatty acids achieved by Y. lipolytica YlR2 was at $0.268 \mathrm{~g} / \mathrm{L}$, and the titer was only 1.7 folds greater than the control on day 7 (Figure 2A). When the growth of the resultant knockout strains checked, no obvious growth defect was observed in the mutant YlR1 (Figure 2B). However, the growth of Y. lipolytica YIR2 was severely affected by blocking pathways for biosynthesis of TAGs and steryl esters (Figure 2B). Both the growth rate and final biomass yield of Y. lipolytica YlR2 were much lower than those of the strain YlR1 and control Po4f Ura. At the end of cultivation, $\mathrm{OD}_{600}$ of cell culture of Y. lipolytica YlR2 was only around half of that of the control strain under the same culture condition (Figure 2B). In contrast, there was no deleterious effect on growth observed in $S$. cerevisiae by the similar genetic manipulations of genes involved in TAG and steryl ester biosynthesis (Sandager et al., 2002; Valle-Rodríguez et al., 2014). Based on the improved cell growth and production performance, strain YIR1 was selected for further improvement of fatty acid production.

\section{Improvement of Fatty Acid Production by Overexpression of Target Genes}

To further enhance production of fatty acids, three genes, including $Y l A c c 1, Y l H x k 1$, and tesA, from E. coli were expressed individually in Y. lipolytica mutant $(\triangle F a a 1, \Delta F a a 2, \Delta F a a 3, \Delta F a t 1$, and $\triangle P x a 1)$. The strain expressing a truncated version of Tes $A$ without signal peptide from $E$. coli produced $2.3 \mathrm{~g} / \mathrm{L}$ fatty acids on day 5, whereas the control strain Po4f only produced $0.198 \mathrm{~g} / \mathrm{L}$ fatty acids under the same culture conditions (Figure 3). The concentration of fatty acids in the strain expressing tes $A$ declined to $1.45 \mathrm{~g} / \mathrm{L}$ on day 7 . The decrease in fatty acid content after day 5 in strain Y. lipolytica YlRX TesA might be due to free fatty acid degradation by other unidentified fatty acyl-CoA synthetases and further utilization for biosynthesis of TAGs and steryl esters when glucose was depleted.

The recombinant $Y$. lipolytica YlRX Acc1 produced fatty acids at the level of 1.57 and $1.65 \mathrm{~g} / \mathrm{L}$ on day 5 and 7 , respectively. 


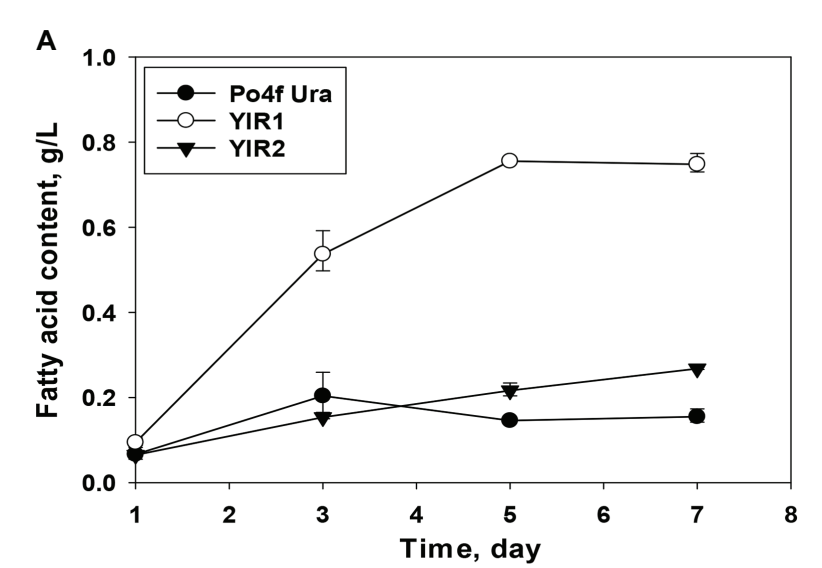

B

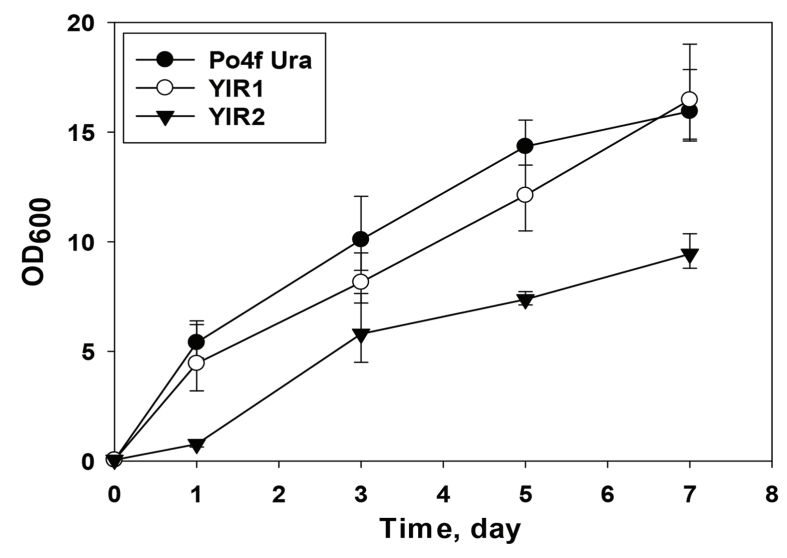

FIGURE 2 | (A) Comparison of fatty acid produced by Y. lipolytica knockout strains including YIR1 ( $\triangle F$ Faa1, $\triangle F$ Fa3, $\triangle F a a 4, \Delta F a t 1$, and $\triangle P$ Pa1) and YIR2 ( $\triangle D$ ga 1, $\triangle D g a 2, \triangle L g a 1$, and $\triangle A r e 1)$, where Po4f Ura was used as a control strain over the culture period of 7 days. (B) Comparison of cell growth of Y. lipolytica strains including Po4f Ura, YIR1, and YIR2. The culture was carried out in flasks at $28^{\circ} \mathrm{C}$ with shaking speed at $180 \mathrm{rpm}$.

The strain YlRX Hexo accumulated $\sim 1 \mathrm{~g} / \mathrm{L}$ free fatty acids on day 5 and 7 . These results showed that expression of TesA from E. coli in Y. lipolytica could improve fatty acid production more significantly than expression of $Y l A c c 1$ and $Y l H x k 1$. The YlHxkl expressed strain showed less improvement in fatty acid accumulation, and this was consistent with the previous report where expression of $Y l H x k 1$ mainly improved lipid production when fructose, instead of glucose, was used as carbon source (Lazar et al., 2014). Extracellular fatty acids secreted by the engineered strains were negligible. There was no obvious growth defect was observed, suggesting high tolerance of Y. lipolytica to long-chain (C16-C18) fatty acids.

\section{Glucose Consumption, Cell Growth, Lipid Production, and Fatty Acid Profiles of Recombinants}

For complete characterization of fatty acid-overproducing strains, glucose consumption, and cell growth during fatty acid accumulation were measured (Figure 4A). The glucose concentration maintained

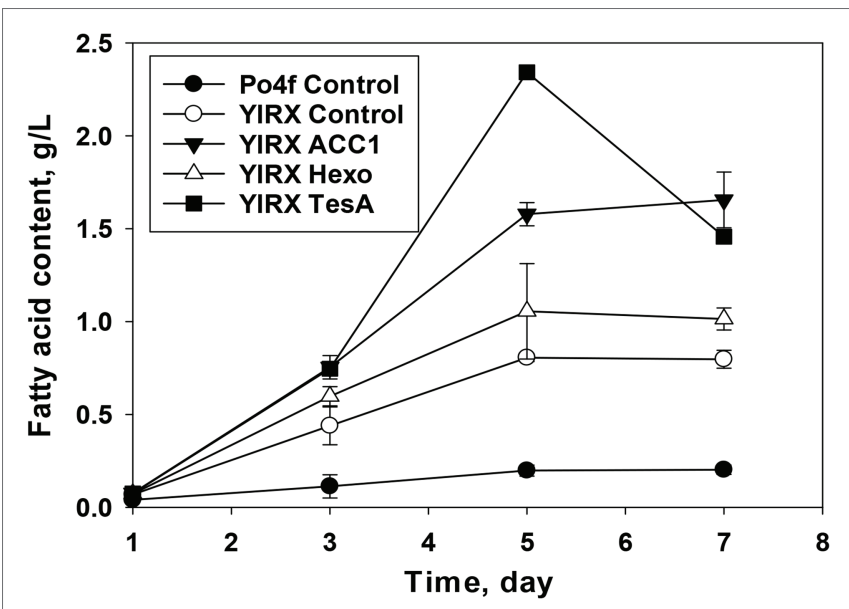

FIGURE 3 | Fatty acid production by $Y$. lipolytica knockout ( $\triangle$ Faa1, $\triangle$ Faa2, $\triangle F a a 3, \triangle F a t 1$, and $\triangle P x a 1)$ overexpressing $Y \mid A c c 1, Y I H \times k 1$, and tesA from Escherichia coli. Y. lipolytica YIRX Control and Po4f Control were cultivated under the same conditions. Cultivation was carried out in a shaker at $28^{\circ} \mathrm{C}$ with shaking speed at $180 \mathrm{rpm}$.

at $80 \mathrm{~g} / \mathrm{L}$ at the early stage of the cultivation of $Y$. lipolytica YlRX TesA. Y. lipolytica expressing tesA gene completely consumed glucose by day 7 , but there was 37.45 and $9.3 \mathrm{~g} / \mathrm{L}$ of residual glucose could be detected in the culture media of Po4f Control and YIRX Control, respectively. It was observed that Y. lipolytica YlRX TesA had higher biomass yield than the other two strains. Neither the strain YlRX Accl nor YlRX Hexo used up glucose at the end of culture period, and there was around $7 \mathrm{~g} / \mathrm{L}$ glucose left in the culture media (Supplementary Figure S1). The reason for growth improvement after expression of TesA is not clear but may be attributed to rapid conversion of inhibitory acyl-CoA molecules such as steroyl-CoA and palmityl-CoA to their counterparts of fatty acids. The results also indicated that fatty acid content in Y. lipolytica YlRX TesA was much higher than the contents in strains Po4f Control and YlRX Control, up to 9.2\% of CDW on day 5 (Figure $4 \mathrm{~B}$ ).

An obvious change in the composition of fatty acids was observed in the recombinant Y. lipolytica YlRX TesA (Figure 5). There was a remarkable increase in saturated fatty acids, including palmitic acid (C16:0) and stearic acid (C18:0), in strain YlRX TesA compared with those in the control strain Po4f. The amount of saturated fatty acids was $\sim 3.5$ times higher than that of unsaturated fatty acids. Heptadecylic acid (C17:0), a very rare metabolite in the cells, was detected. In the strain engineered for overproduction of lipids, production of heptadecylic acid was also observed, perhaps, due to biosynthesis of less favored odd-chained fatty acid resulted from active lipogenesis (Blazeck et al., 2014). In addition, high lipid titers also allowed measurement and detection of fatty acids with low abundance.

Regarding the accumulation of lipids in the recombinants developed in this study, Y. lipolytica YIRX TesA reached the highest lipid content of $4.64 \mathrm{~g} / \mathrm{L}$ on day 7 (Figure 6). Lipid concentration increased in all the modified strains compared with the parent strain. The significant decrease of fatty acid content but an increase in lipid content was observed on 

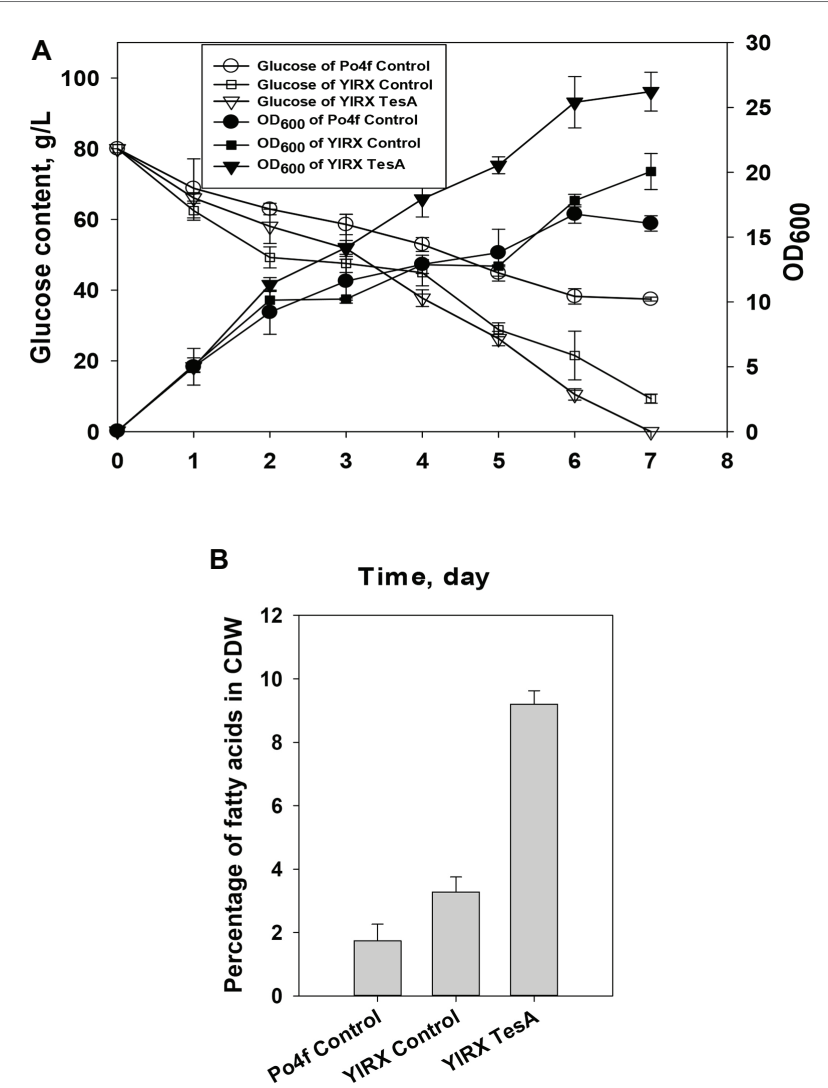

FIGURE 4 | (A) Time function of cell growth and glucose consumption of Y. lipolytica strains including Po4f Control, YIRX Control, and YIRX TesA, and (B) Fatty acid content in Y. lipolytica Po4f Control, YIRX TesA, and YIRX Control grown for 5 days on the basis of cell dry weight (CDW) (wt/wt).

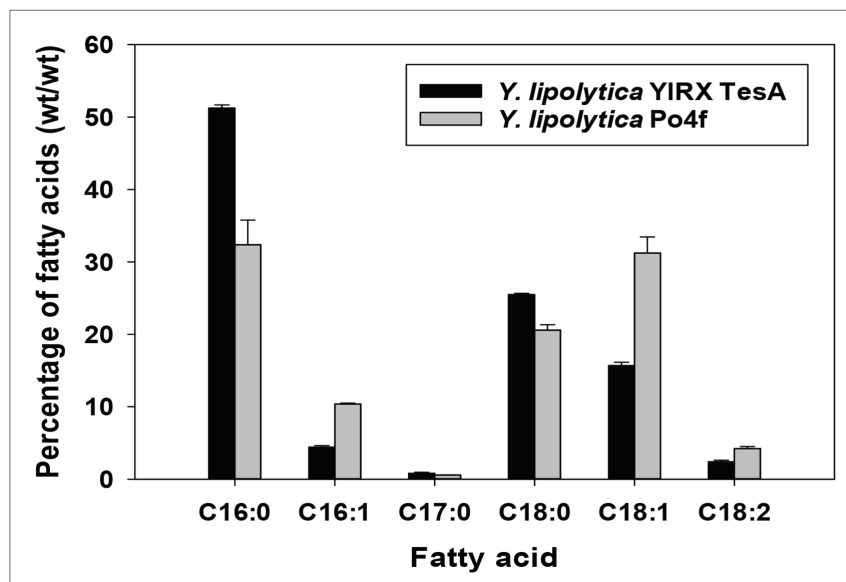

FIGURE 5 | Profile of fatty acid in Y. lipolytica YIRX TesA and control strain Po4f cultured for 5 days. The fatty acid composition profile was shown as percent (wt/wt). The carbon source is indicated in parentheses after the strain name. C16:0, palmitic acid; C16:1, palmitoleic acid; C17:0, heptadecylic acid; C18:0, stearic acid; C18:1, oleic acid; and C18:2, linoleic acids. Fatty acids were quantified using external standards with gas chromatography (GC) analysis.

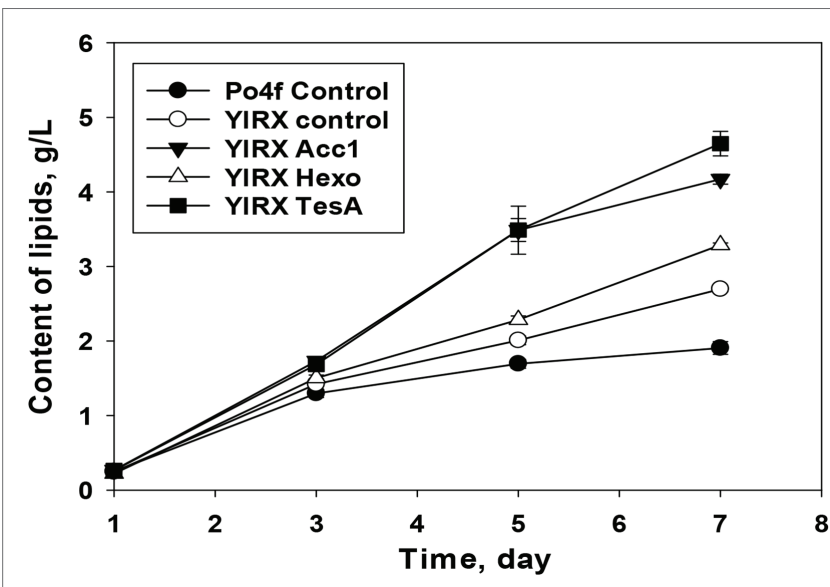

FIGURE 6 | Time function of lipid accumulation in the developed recombinant strains overexpressing $Y \mid A c c 1, Y I H \times k 1$, and tesA from E. coli. Y. lipolytica Po4f Control and YIRX Control were also cultured under the same conditions. Lipids consisted of free fatty acids, TAGs, steryl esters, and other intermediates containing fatty acid group.

day 7 in Y. lipolytica YlRX TesA, suggesting rapid turnover between fatty acids and lipids. The similar pattern for interconversion of fatty acids into lipids was not observed in the strain YlRX Control (Supplementary Figure S2). Both YlRX Acc1 and YlRX Hexo accumulated more lipids than the control strain and the lipid content reached 4.17 and $3.2 \mathrm{~g} / \mathrm{L}$, respectively (Figure 6). These results demonstrated that Y. lipolytica had a great potential to accumulate fatty acids and other derivates.

\section{DISCUSSION}

Results from this study demonstrated the feasibility of metabolic engineering of oleaginous yeast, Y. lipolytica, to overproduce fatty acids. The strains were modified by rewiring lipid metabolism to produce long-chain fatty acids as the targeted products. The major metabolic engineering approaches included impairing fatty acid activation and degradation pathways and expression of bacterial thioesterase for hydrolysis of acyl-CoA into fatty acids. The maximum fatty acid titer of $2.3 \mathrm{~g} / \mathrm{L}$ was achieved under a shake flask culture condition. The developed strain showed a substantial increase in saturated fatty acids. This property is potentially beneficial for production of biodiesel due to better oxidative stability and higher combustion value of FAMEs consisting of saturated fatty acids than unsaturated fatty acids (Rasoul-Amini et al., 2011). Although the produced fatty acids were mainly accumulated in the cells without being secreted, there was no growth defect observed. Aligned with our work for metabolic engineering of Y. lipolytica to produce long-chain dicarboxylic acid (Abghari et al., 2017) and fatty alcohol (Wang et al., 2016), the current study further improved Y. lipolytica for production of long-chain (C16-C18) fatty acids.

This study explored Y. lipolytica's ability of overproduction of fatty acids. Two main strategies, including deletion of $\mathrm{faa}$ genes for blocking fatty acid consumption and deletion of genes related to assembly of TAGs and steryl esters, have been 
implemented prior to overexpression of genes, including $Y l H x k 1$, YlAcc1, and TesA. It was observed that the strategy of blocking activation and peroxisomal uptake of fatty acids worked better than the elimination of TAG and steryl ester biosynthesis in terms of improving fatty acid production. Blocking TAG and steryl ester biosynthesis in Y. lipolytica led to the detrimental effects on cell growth, although these pathways were presented as non-essential pathways in S. cerevisiae (Sandager et al., 2002; Valle-Rodríguez et al., 2014). This result was consistent with the previous findings of deletion of the genes for biosynthesis of TAGs and steryl esters in Y. lipolytica (Beopoulos et al., 2012; Ledesma-Amaro et al., 2016). Expression of thioesterase from E. coli for hydrolysis of acyl-CoA into fatty acids further improved the cell growth of the strain bearing disrupted faa and pxal genes. These results suggested that the inhibition to the cell growth was not mainly attributed to fatty acids but acyl-CoA accumulated in the cells. Although four candidate faa genes from Y. lipolytica were identified based on their identities to the corresponding genes from $S$. cerevisiae, two of them were not fully characterized for their biochemical roles in fatty acid utilization (Dulermo et al., 2015). The function of FAA enzymes can be evaluated by comparison of growth on fatty acids with different chain lengths, lipid and fatty acid contents, and enzymatic activities of individual and combinational knockouts bearing disrupted faa genes. Alternatively, the substrate preference of FAA enzyme can be investigated by expression of the targeted gene in a $S$. cerevisiae mutant deficient of all the native faa genes and observation of the growth of recombinant bearing a specific faa gene from Y. lipolytica on fatty acids with different chain lengths (Knoll et al., 1995). It is also important to understand the relationship of the transcriptional regulation of $f a a$ genes between lipogeneses (Liu et al., 2019). The localization of FAA enzymes in cellular compartments such as cytosol and peroxisome can be tracked by using fluorescent protein tagging (Dulermo et al., 2016; Bredeweg et al., 2017). Importantly, compartmentalization of enzymes for biosynthesis of lipid-related compounds to the yeast organelles such as peroxisome and endoplasmic reticulum instead of cytosol was beneficial to achieve higher titers and yields for production of oleochemical including fatty acids (Xu et al., 2016; Yang et al., 2019).

As an oleaginous yeast, Y. lipolytica is uniquely advantageous for production of fatty acids and fatty acid-based chemicals by capitalizing on advancements in synthetic biology. The results in this study provide insights into improvement of Y. lipolytica for fatty acid production by metabolic engineering. The results add new information and advance the current understanding of mechanism underlying fatty acid and lipid metabolism. Although the oleaginous yeast Y. lipolytica can generate a high

\section{REFERENCES}

Abghari, A., Madzak, C., and Chen, S. (2017). Combinatorial engineering of Yarrowia lipolytica as a promising cell biorefinery platform for the de novo production of multi-purpose long chain dicarboxylic acids. Fermentation 3:40. doi: 10.3390/fermentation 3030040

Adrio, J. L. (2017). Oleaginous yeasts: promising platforms for the production of oleochemicals and biofuels. Biotechnol. Bioeng. 114, 1915-1920. doi: 10.1002/bit.26337 carbon flux toward cytosolic acetyl-CoA under nitrogen limiting culture conditions, increasing acetyl-CoA pool further improved oleochemical production (Xu et al., 2016). The performance of the strains developed in this study needs to be further improved. The future work will include pathway optimization and evolution of the stains for target production, optimization of the cultivation conditions including fed-batch cultivation by using bioreactor, expression of genes encoding efflux pumps for secretion of produced fatty acids to the supernatant (Doshi et al., 2013), and manipulation of regulatory factors governing fatty acid and lipid biosynthesis (Seip et al., 2013).

\section{DATA AVAILABILITY STATEMENT}

The raw data supporting the conclusions of this article will be made available by the authors, without undue reservation.

\section{AUTHOR CONTRIBUTIONS}

XX conceived the idea, and XX and SC supervised the work. $\mathrm{XX}$ and RG planned the experiments and conducted the experiments including molecular cloning and construction of yeast recombinants. RG collected and analyzed the data. All authors contributed to the article and approved the submitted version.

\section{FUNDING}

This work was supported by the USDA National Institute of Food and Agriculture, McIntire-Stennis project WNP00009.

\section{ACKNOWLEDGMENTS}

The authors would like to thank Washington State University for supporting this research. We gratefully acknowledge Dr. Guokun Wang, Dr. Yonghong Meng, and Dr. Ali Abghari (Department of Biological Systems Engineering, Washington State University) for critical discussions.

\section{SUPPLEMENTARY MATERIAL}

The Supplementary Material for this article can be found online at: https://www.frontiersin.org/articles/10.3389/fmicb.2020.01717/ full\#supplementary-material. 
Bredeweg, E. L., Pomraning, K. R., Dai, Z., Nielsen, J., Kerkhoven, E. J., and Baker, S. E. (2017). A molecular genetic toolbox for Yarrowia lipolytica. Biotechnol. Biofuels 10:2. doi: 10.1186/s13068-016-0687-7

Cao, X., Lv, Y. -B., Chen, J., Imanaka, T., Wei, L. -J., and Hua, Q. (2016). Metabolic engineering of oleaginous yeast Yarrowia lipolytica for limonene overproduction. Biotechnol. Biofuels 9:214. doi: 10.1186/s13068-016-0626-7

Chen, L., Zhang, J., Lee, J., and Chen, W. N. (2014). Enhancement of free fatty acid production in Saccharomyces cerevisiae by control of fatty acyl-CoA metabolism. Appl. Microbiol. Biotechnol. 98, 6739-6750. doi: 10.1007/ s00253-014-5758-8

Coe, N. R., Smith, A. J., Frohnert, B. I., Watkins, P. A., and Bernlohr, D. A. (1999). The fatty acid transport protein (FATP1) is a very long chain acyl-CoA synthetase. J. Biol. Chem. 274, 36300-36304. doi: 10.1074/jbc.274.51.36300

Cui, Z., Gao, C., Li, J., Hou, J., Lin, C. S. K., and Qi, Q. (2017). Engineering of unconventional yeast Yarrowia lipolytica for efficient succinic acid production from glycerol at low pH. Metab. Eng. 42, 126-133. doi: 10.1016/j.ymben.2017.06.007

Dai, Z., Huang, M., Chen, Y., Siewers, V., and Nielsen, J. (2018). Global rewiring of cellular metabolism renders Saccharomyces cerevisiae Crabtree negative. Nat. Commun. 9:3059. doi: 10.1038/s41467-018-05409-9

Doshi, R., Nguyen, T., and Chang, G. (2013). Transporter-mediated biofuel secretion. Proc. Natl. Acad. Sci. U. S. A. 110, 7642-7647. doi: 10.1073/ pnas. 1301358110

Dulermo, R., Gamboa-Meléndez, H., Dulermo, T., Thevenieau, F., and Nicaud, J. -M. (2014). The fatty acid transport protein Fatlp is involved in the export of fatty acids from lipid bodies in Yarrowia lipolytica. FEMS Yeast Res. 14, 883-896. doi: 10.1111/1567-1364.12177

Dulermo, R., Gamboa-Meléndez, H., Ledesma-Amaro, R., Thévenieau, F., and Nicaud, J. -M. (2015). Unraveling fatty acid transport and activation mechanisms in Yarrowia lipolytica. Biochim. Biophys. Acta Mol. Cell Biol. Lipids 1851, 1202-1217. doi: 10.1016/j.bbalip.2015.04.004

Dulermo, R., Gamboa-Meléndez, H., Ledesma-Amaro, R., Thevenieau, F., and Nicaud, J. -M. (2016). Yarrowia lipolytica AAL genes are involved in peroxisomal fatty acid activation. Biochim. Biophys. Acta Mol. Cell Biol. Lipids 1861, 555-565. doi: 10.1016/j.bbalip.2016.04.002

Faergeman, N., Black, P., Zhao, X., Knudsen, J., and DiRusso, C. (2001). The acyl-CoA synthetases encoded within FAA1 and FAA4 in Saccharomyces cerevisiae function as components of the fatty acid transport system linking import, activation, and intracellular utilization. J. Biol. Chem. 276, 37051-37059. doi: $10.1074 /$ jbc.M100884200

Ferreira, R., Teixeira, P. G., Gossing, M., David, F., Siewers, V., and Nielsen, J. (2018). Metabolic engineering of Saccharomyces cerevisiae for overproduction of triacylglycerols. Metab. Eng. Commun. 6, 22-27. doi: 10.1016/j. meteno.2018.01.002

Folch, J., Lees, M., and Stanley, G. H. S. (1957). A simple method for the isolation and purification of total lipides from animal tissues. J. Biol. Chem. 226, 497-509.

Gajdoš, P., Ledesma-Amaro, R., Nicaud, J. -M., Čertík, M., and Rossignol, T. (2016). Overexpression of diacylglycerol acyltransferase in Yarrowia lipolytica affects lipid body size, number and distribution. FEMS Yeast Res. 16:fow062. doi: $10.1093 /$ femsyr/fow062

Knoll, L. J., Johnson, D. R., and Gordon, J. (1995). Complementation of Saccharomyces cerevisiae strains containing fatty acid activation gene (FAA) deletions with a mammalian acyl-CoA synthetase. J. Biol. Chem. 270, 10861-10867. doi: $10.1074 / \mathrm{jbc} .270 .18 .10861$

Koch, B., Schmidt, C., and Daum, G. (2014). Storage lipids of yeasts: a survey of nonpolar lipid metabolism in Saccharomyces cerevisiae, Pichia pastoris, and Yarrowia lipolytica. FEMS Microbiol. Rev. 38, 892-915. doi: 10.1111/1574-6976.12069

Lazar, Z., Dulermo, T., Neuvéglise, C., Crutz-Le Coq, A. -M., and Nicaud, J. -M. (2014). Hexokinase-a limiting factor in lipid production from fructose in Yarrowia lipolytica. Metab. Eng. 26, 89-99. doi: 10.1016/j.ymben.2014.09.008

Leber, C., Polson, B., Fernandez-Moya, R., and Da Silva, N. A. (2015). Overproduction and secretion of free fatty acids through disrupted neutral lipid recycle in Saccharomyces cerevisiae. Metab. Eng. 28, 54-62. doi: 10.1016/j. ymben.2014.11.006

Ledesma-Amaro, R., Dulermo, R., Niehus, X., and Nicaud, J. -M. (2016). Combining metabolic engineering and process optimization to improve production and secretion of fatty acids. Metab. Eng. 38, 38-46. doi: 10.1016/j. ymben.2016.06.004
Liu, H., Marsafari, M., Deng, L., and Xu, P. (2019). Understanding lipogenesis by dynamically profiling transcriptional activity of lipogenic promoters in Yarrowia lipolytica. Appl. Microbiol. Biotechnol. 103, 3167-3179. doi: 10.1007/ s00253-019-09664-8

Liu, L., Pan, A., Spofford, C., Zhou, N., and Alper, H. S. (2015). An evolutionary metabolic engineering approach for enhancing lipogenesis in Yarrowia lipolytica. Metab. Eng. 29, 36-45. doi: 10.1016/j.ymben.2015.02.003

Liu, D., Wan, N., Zhang, F., Tang, Y. J., and Wu, S. G. (2017). Enhancing fatty acid production in Escherichia coli by Vitreoscilla hemoglobin overexpression. Biotechnol. Bioeng. 114, 463-467. doi: 10.1002/bit.26067

Madzak, C. (2018). Engineering Yarrowia lipolytica for use in biotechnological applications: a review of major achievements and recent innovations. Mol. Biotechnol. 60, 621-635. doi: 10.1007/s12033-018-0093-4

Markham, K. A., Palmer, C. M., Chwatko, M., Wagner, J. M., Murray, C., Vazquez, S., et al. (2018). Rewiring Yarrowia lipolytica toward triacetic acid lactone for materials generation. Proc. Natl. Acad. Sci. U. S. A. 115, 2096-2101. doi: 10.1073/pnas.1721203115

Qiao, K., Wasylenko, T. M., Zhou, K., Xu, P., and Stephanopoulos, G. (2017). Lipid production in Yarrowia lipolytica is maximized by engineering cytosolic redox metabolism. Nat. Biotechnol. 35, 173-177. doi: 10.1038/nbt.3763

Rasoul-Amini, S., Montazeri-Najafabady, N., Mobasher, M. A., Hoseini-Alhashemi, S., and Ghasemi, Y. (2011). Chlorella sp.: a new strain with highly saturated fatty acids for biodiesel production in bubble-column photobioreactor. Appl. Energy 88, 3354-3356. doi: 10.1016/j.apenergy.2010.12.040

Sandager, L., Gustavsson, M. H., Stahl, U., Dahlqvist, A., Wiberg, E., Banas, A., et al. (2002). Storage lipid synthesis is non-essential in yeast. J. Biol. Chem. 277, 6478-6482. doi: 10.1074/jbc.M109109200

Seip, J., Jackson, R., He, H., Zhu, Q., and Hong, S. -P. (2013). Snf1 is a regulator of lipid accumulation in Yarrowia lipolytica. Appl. Environ. Microbiol. 79, 7360-7370. doi: 10.1128/AEM.02079-13

Shockey, J., Schnurr, J., and Browse, J. (2000). Characterization of the AMP-binding protein gene family in Arabidopsis thaliana: will the real acyl-CoA synthetases please stand up? Biochem. Soc. Trans. 28, 955-957. doi: 10.1042/bst0280955

Steen, E. J., Kang, Y., Bokinsky, G., Hu, Z., Schirmer, A., Mcclure, A., et al. (2010). Microbial production of fatty-acid-derived fuels and chemicals from plant biomass. Nature 463, 559-562. doi: 10.1038/nature08721

Tai, M., and Stephanopoulos, G. (2013). Engineering the push and pull of lipid biosynthesis in oleaginous yeast Yarrowia lipolytica for biofuel production. Metab. Eng. 15, 1-9. doi: 10.1016/j.ymben.2012.08.007

Valle-Rodríguez, J. O., Shi, S., Siewers, V., and Nielsen, J. (2014). Metabolic engineering of Saccharomyces cerevisiae for production of fatty acid ethyl esters, an advanced biofuel, by eliminating non-essential fatty acid utilization pathways. Appl. Energy 115, 226-232. doi: 10.1016/j.apenergy.2013.10.003

Wang, G., Xiong, X., Ghogare, R., Wang, P., Meng, Y., and Chen, S. (2016). Exploring fatty alcohol-producing capability of Yarrowia lipolytica. Biotechnol. Biofuels 9:107. doi: 10.1186/s13068-016-0512-3

Wang, J., Zhang, B., and Chen, S. (2011). Oleaginous yeast Yarrowia lipolytica mutants with a disrupted fatty acyl-CoA synthetase gene accumulate saturated fatty acid. Process Biochem. 46, 1436-1441. doi: 10.1016/j.procbio.2011.03.011

Xiong, X., Lian, J., Yu, X., Garcia-Perez, M., and Chen, S. (2016). Engineering levoglucosan metabolic pathway in Rhodococcus jostii RHA1 for lipid production. J. Ind. Microbiol. Biotechnol. 43, 1551-1560. doi: 10.1007/ s10295-016-1832-9

Xiong, X., Wang, X., and Chen, S. (2012). Engineering of a xylose metabolic pathway in Rhodococcus strains. Appl. Environ. Microbiol. 78, 5483-5491. doi: 10.1128/AEM.08022-11

Xu, P., Qiao, K., Ahn, W. S., and Stephanopoulos, G. (2016). Engineering Yarrowia lipolytica as a platform for synthesis of drop-in transportation fuels and oleochemicals. Proc. Natl. Acad. Sci. U. S. A. 113, 10848-10853. doi: $10.1073 /$ pnas. 1607295113

Yan, Q., and Pfleger, B. F. (2020). Revisiting metabolic engineering strategies for microbial synthesis of oleochemicals. Metab. Eng. 58, 35-46. doi: 10.1016/j. ymben.2019.04.009

Yang, K., Qiao, Y., Li, F., Xu, Y., Yan, Y., Madzak, C., et al. (2019). Subcellular engineering of lipase dependent pathways directed towards lipid related organelles for highly effectively compartmentalized biosynthesis of triacylglycerol derived products in Yarrowia lipolytica. Metab. Eng. 55, 231-238. doi: 10.1016/j.ymben.2019.08.001 
Zhou, Y. J., Buijs, N. A., Zhu, Z., Qin, J., Siewers, V., and Nielsen, J. (2016). Production of fatty acid-derived oleochemicals and biofuels by synthetic yeast cell factories. Nat. Commun. 7:11709. doi: 10.1038/ncomms11709

Conflict of Interest: The authors declare that the research was conducted in the absence of any commercial or financial relationships that could be construed as a potential conflict of interest.
Copyright (c) 2020 Ghogare, Chen and Xiong. This is an open-access article distributed under the terms of the Creative Commons Attribution License (CC BY). The use, distribution or reproduction in other forums is permitted, provided the original author(s) and the copyright owner(s) are credited and that the original publication in this journal is cited, in accordance with accepted academic practice. No use, distribution or reproduction is permitted which does not comply with these terms. 